\title{
Localization in disordered media, anomalous roughening, and coarsening dynamics of faceted surfaces
}

\author{
Ivan G. Szendro, ${ }^{1,2, *}$ Juan M. López, ${ }^{1, \dagger}$ and Miguel A. Rodríguez ${ }^{1, \ddagger}$ \\ ${ }^{1}$ Instituto de Física de Cantabria (IFCA), CSIC-UC, E-39005 Santander, Spain \\ ${ }^{2}$ Departamento de Física Moderna, Universidad de Cantabria, Avenida Los Castros, E-39005 Santander, Spain
}

(Received 26 March 2007; revised manuscript received 30 May 2007; published 20 July 2007)

\begin{abstract}
We study a surface growth model related to the Kardar-Parisi-Zhang equation for nonequilibrium kinetic roughening, but where the thermal noise is replaced by a static columnar disorder $\eta(\mathbf{x})$. This model is one of the many representations of the problem of particle diffusion in trapping or amplifying disordered media. We find that probability localization in the latter translates into facet formation in the equivalent surface growth problem. Coarsening of the pattern can therefore be identified with the diffusion of the localization center. The emergent faceted structure gives rise to nontrivial scaling properties, including anomalous surface roughening in excellent agreement with an existing conjecture for kinetic roughening of faceted surfaces. In a wider context, our study sheds light onto the scaling properties in other systems displaying this kind of patterned surface.
\end{abstract}

DOI: 10.1103/PhysRevE.76.011603

PACS number(s): 81.15.Aa, 05.40.- a, 64.60.Ht, 89.75.Kd

\section{INTRODUCTION}

Diffusion of a classical field in the presence of random spatially distributed traps and sources is an important topic in contemporary nonequilibrium statistical physics. Through various mathematically equivalent formulations, the problem can be related to a number of nonequilibrium processes, including localization of random particles in disordered media, chemical reactions, directed polymers in random potentials, and stochastic growth models, to mention just a few important examples (see, for instance, [1] and references within). Spatiotemporal properties of transport in trapping and amplifying disordered media were first studied by Zhang [2] and Ebeling et al. $[3,4]$. They considered the problem of a probability density $\phi(\mathbf{x}, t)$ obeying a simple multiplicative stochastic dynamics

$$
\partial_{t} \phi=\nu \nabla^{2} \phi+\eta(\mathbf{x}) \phi(\mathbf{x}, t),
$$

where the random field $\eta(\mathbf{x})$ is Gaussian with zero mean and correlator

$$
\left\langle\eta(\mathbf{x}) \eta\left(\mathbf{x}^{\prime}\right)\right\rangle=\sigma^{2} \delta_{a}\left(\mathbf{x}-\mathbf{x}^{\prime}\right),
$$

where the $\delta$ function smears over some microscopic distance a. Simple dimensional arguments give a characteristic length scale $\ell_{0} \sim(\nu / \sigma)^{2 /(4-d)}$ associated with the scales above which the behavior is dominated by the disorder (strong-coupling limit). Some exact results for this model have been obtained for the asymptotic behavior of $\ln \langle\phi(\mathbf{x}, t)\rangle[5]$, where $\langle\cdots\rangle$ is an average over disorder realizations. However, $\langle\ln \phi(\mathbf{x}, t)\rangle$, rather than $\langle\phi(\mathbf{x}, t)\rangle$ itself, is the quantity of interest.

This simple model has multiple connections with other important disordered systems [1]. In particular, the Green propagator $\phi(\mathbf{x}, t ; \mathbf{y}, 0)$ of Eq. (1), i.e., the solution for the

\footnotetext{
*szendro@ifca.unican.es

†lopez@ifca.unican.es

†rodrigma@ifca.unican.es
}

initial condition $\phi(\mathbf{x}, 0)=\delta(\mathbf{x}-\mathbf{y})$, can be interpreted as the restricted partition function of the $d$-dimensional Gaussian polymer of length $t$ at finite temperature with one end at position $\mathbf{y}$ and the other end at position $\mathbf{x}$, or, equivalently, as a $(d+1)$-directed polymer in a random potential uncorrelated in the $t$ direction. In the zero-temperature limit the problem can be formulated in terms of an optimal path [1].

One can qualitatively analyze the type of solutions of Eq. (1). It is interesting to notice that the multiplicative character of Eq. (1) leads to sharply localized solutions around random centers [2]. Given a single realization of the random potential $\eta(\mathbf{x})$, the growth of $\phi(\mathbf{x}, t)$ is characterized by strong spatial fluctuations because the asymptotic behavior is concentrated in several peaks which grow exponentially in time. The localization centers correspond to space positions $\mathbf{x}_{c}$ where the potential $\eta(\mathbf{x})$ randomly takes large positive values. There is an exponential profile around such a typical center, $\exp \left(-\left|\mathbf{x}-\mathbf{x}_{c}\right| / \xi\right)$, with a certain localization length $\xi$ reaching out from the center $\mathbf{x}_{c}$. However, for long enough time, a new and better center will appear, which over time will outgrow the previous center, and the field $\phi(\mathbf{x}, t)$ will then concentrate around the new attractor, and so on. This hopping from center to center will continue forever, with longer and longer times spent at each new localization center. There has been considerable theoretical discussion about the time dependence of the position of the dominant localization center [2-4]. Theoretical studies based on replica scaling analysis [6] and Flory-like approaches [8-10] have finally concluded that the dynamics of the strongest localization center in the strong-coupling regime is weakly subballistic, $x_{c}(t) \sim t / \mathrm{ln}^{3 / 4} t$, in any dimension.

Further insight can be achieved by the nonlinear HopfCole transformation $\phi(\mathbf{x}, t)=\exp [\lambda h(\mathbf{x}, t) / \nu]$, which leads us to

$$
\partial_{t} h(\mathbf{x}, t)=\nu \nabla^{2} h+\lambda(\nabla h)^{2}+\eta(\mathbf{x}),
$$

describing the dynamics of the surface $h(\mathbf{x}, t)$ in a quenched columnar disorder with short-ranged correlations 
$\left\langle\eta(\mathbf{x}, t) \eta\left(\mathbf{x}^{\prime}, t\right)\right\rangle=2 D \delta_{a}\left(\mathbf{x}-\mathbf{x}^{\prime}\right)$, where $2 D=(\nu \sigma / \lambda)^{2}$. In the interface growth problem, we are interested in the roughening properties of an initially flat interface; we are therefore interested in the initial condition $h(\mathbf{x}, 0)=0$, which in the polymer picture can be interpreted as the problem of a polymer of length $t$ with one end at $\mathbf{x}$ and the other end at any position, $\phi(\mathbf{x}, t)=\int d \mathbf{y} \phi(\mathbf{x}, t ; \mathbf{y}, 0)$. The interface height is then the free energy of the polymer, $h(\mathbf{x}, t)=\ln \phi(\mathbf{x}, t)=$ $-\mathcal{F}(\mathbf{x}, t)$, with the opposite sign.

This equation is a close relative of the celebrated KardarParisi-Zhang (KPZ) equation for scale-invariant surface growth [7], but with the usual thermal noise $\eta(\mathbf{x}, t)$ replaced by a static columnar disorder $\eta(\mathbf{x})$. It has been pointed out [8] that at large length scales the interface is dominated by the disorder values at single sites, such that the central limit theorem does not apply, and the specific choice for the disorder distribution may be relevant. This is particularly relevant for polymers at zero temperature, where the disorder microscopic details do affect the form of the diffusion law in a highly nontrivial way [8]. Equation (3) has also been studied in the context of directed and undirected polymers in the presence of columnar disorder [8-10]. This type of columnar disorder has attracted much interest in theoretical studies of the pinning of magnetic flux lines in high-temperature superconductors [11-15], where it can be induced experimentally by ion radiation [16].

In this paper, we study the scaling properties of a surface following Eq. (3). We shall show that localization of the solutions of Eq. (1) naturally leads to a faceted structure of the corresponding surface $h(\mathbf{x}, t)$ in Eq. (3). We observe that these facets coarsen with a coarsening length directly related to the motion of the localization center $x_{c}(t)$ in the original problem. This is in agreement with earlier Flory-type arguments [8-10]. Our main result concerns our finding of anomalous scaling properties of the surface, in excellent agreement with an earlier conjecture by Ramasco et al. [17] for kinetically rough faceted surfaces. In the more general framework of surface and thin film growth models, our study provides an example of the interplay between static disorder and nonlinear terms for the production of self-organized faceted surfaces.

The paper is organized as follows. In Sec. II, we present numerical results, which show the existence of faceted patterns and coarsening in $d=1$. We also discuss the interpretation of these results in the context of localization in random media. In Sec. III, kinetic roughening is studied numerically in $d=1,2$. We shall need anomalous scaling ideas to understand the scaling properties of the surface, which are much more involved than those captured by the renormalization approach. In Sec. IV, we take a closer look at the surface and show that it can be decomposed into a base pattern plus some fluctuations. Both components can be studied independently, and we show that their dynamics allows understanding of the anomalous scaling properties observed. We finish with some concluding remarks in Sec. V.

\section{FACETED GROWTH AND COARSENING}

In order to perform a numerical integration of Eq. (3), the parameters can easily be rescaled to have only one indepen-

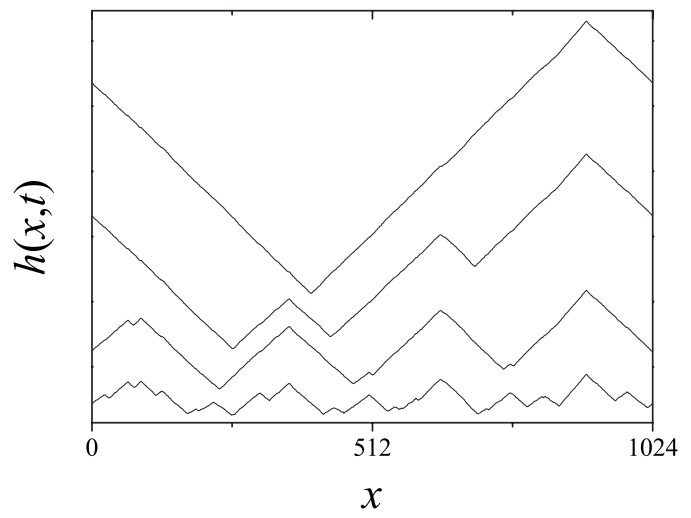

FIG. 1. Typical evolution of a surface obeying (3) in a system of size $L=1024$. From bottom to top, the curves correspond to times $t / \Delta t=2^{13}, 2^{15}, 2^{17}, 2^{19}$ and are shifted for the sake of clarity.

dent control parameter-namely, the coupling constant $\bar{\lambda}$ $=\lambda \sqrt{D / \nu^{4}}$. Note that this coupling constant is related to the characteristic length scale in Eq. $(1), \bar{\lambda}=\ell_{0}^{2 /(d-4)} / \sqrt{2}$. This implies that for $d<4$ the strong-coupling behavior $\left(\ell_{0} \ll a\right)$ of the polymer problem corresponds to that of the interface in the strong-coupling regime $(\bar{\lambda} \gg 1)$.

We have used a standard finite-differences scheme as follows:

$$
\begin{aligned}
h(i, t+\Delta t)= & h(i, t)+\Delta t \eta(i)+\Delta t(\bar{\lambda} / 4)[h(i+1, t)-h(i-1, t)]^{2} \\
& +\Delta t[h(i+1, t)+h(i-1, t)-2 h(i, t)],
\end{aligned}
$$

where the lattice spacing has been set to unity. We start our simulation from a uniformly flat initial condition $h(i, t=0)$ $=\operatorname{ran}[0,1]$, and periodic boundary conditions $h(0, t)=h(L, t)$ and $h(L+1, t)=h(1, t)$ are imposed at each time step. Simulations with different time steps were carried out, and the scheme proved to be stable and well behaved for a time step $\Delta t=0.01$ (or smaller) for the range of parameters simulated. The static disorder $\eta(i)$ is spatially uncorrelated and drawn at random from a normal distribution $\mathcal{N}(0,1)$.

A typical one-dimensional growth pattern of the surface is shown in Fig. 1 for $\bar{\lambda}=2$ in a system size $L=1024$. One observes that the interface organizes itself into a faceted (triangular) pattern with a characteristic slope. The typical lateral size of a triangular mound grows, and, eventually, one ends up with a single large triangle spanning the whole system. Therefore, in a finite-size system, the asymptotic solution consists of a surface growing at constant velocity, conserving its triangular shape. The faceted pattern emerges as an overall long-wavelength behavior resulting from the existence of strong pinning and depinning centers, which in turn arise from the quenched columnar disorder $\eta(x)$. At any given time the surface is then made by superposition of triangular-shape base functions $h\left(x_{0}\right)-s(t)\left|x-x_{0}\right|$, centered around the strong depinning centers $x_{0}$ (i.e., the cusps in Fig. 1 ), where the disorder $\eta(x)$ is large and positive. The evolution was previously described in Refs. [9,10].

The average surface slope $\left\langle\overline{(\nabla h)^{2}}\right\rangle^{1 / 2}$ and the surface velocity $v(t)=\left\langle\overline{\partial_{t} h}\right\rangle$, after spatial averaging in (3), are found to 


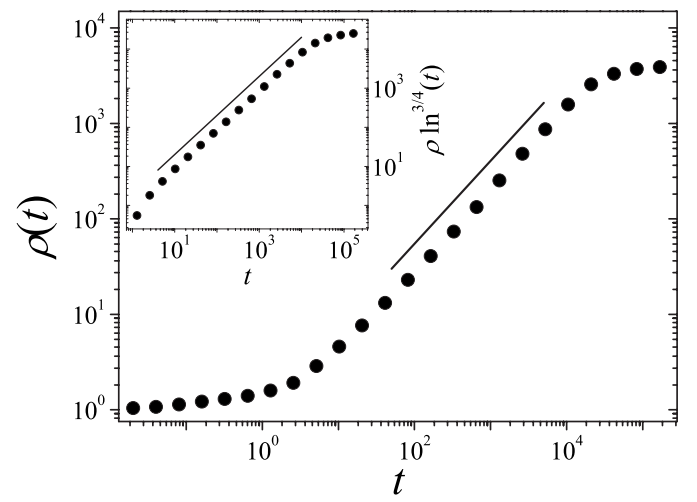

FIG. 2. Evolution of lateral mound size $\rho(t)$. The straight line is plotted to guide the eyes and has slope $1 / z_{\text {coarse }}=-0.87$. The inset shows the sub-ballistic coarsening law by plotting $\rho(t) \ln ^{3 / 4}(t)$ vs time (circles). The solid line is plotted to guide the eye and has slope 1.

satisfy the typical KPZ relation $\left\langle\overline{(\nabla h)^{2}}\right\rangle^{1 / 2}=[v(t) / \bar{\lambda}]^{1 / 2}$. However, it is worth noting here that, in contrast with the thermal $\mathrm{KPZ}$ model, the average interface velocity is not constant in the intermediate-time regime before saturation. We shall discuss this point in more detail in Sec. IV. Only at very long times, when the pattern becomes stationary, does $v(t)$ $\rightarrow$ const, and we expect a limiting triangular profile with a time-independent average slope proportional to $(v(\infty) / \bar{\lambda})^{1 / 2}$.

The coarsening of the surface pattern to larger and larger triangles is commonly characterized by the growth law of the lateral mound size $\rho(t)$. We expect the slope-slope correlation $\langle\overline{\nabla h(x, t) \nabla h(x+r, t)}\rangle$ to decay to zero over a typical distance $\rho(t)$. We measured the coarsening length $\rho(t)$ from the correlation decay plot and the results are shown in Fig. 2. In principle, the coarsening length time behavior can be nicely fitted with a power law $\rho(t) \sim t^{1 / z_{\text {coarse }}}$ with exponent $1 / z_{\text {coarse }}=0.87 \pm 0.03$. However, on theoretical grounds $[8,10]$ we expect the correct scaling to follow a sub-ballistic law $\rho(t) \sim t / \ln ^{3 / 4} t$.

Interestingly, the formation of facets in Eq. (3) is directly related to the localization of the solutions $\phi$ in the multiplicative diffusion equation (1). Note that a facet of slope $s$, $h(x, t)=h\left(x_{0}\right)-s(t)\left|x-x_{0}\right|$, with a cusp at $x=x_{0}$, corresponds to an exponential profile $\phi \propto \exp \left(-s(t)\left|x-x_{0}\right|\right)$, spatially localized around $x_{0}$ for the particle probability density in (1). In addition, the lateral mound size $\rho(t)$ corresponds to the average distance separating one localization center from the next one, and therefore the coarsening growth law is to be compared with the sub-ballistic diffusion of the localization center $\sim t / \ln ^{3 / 4} t$ in Refs. [8,10].

\section{FACETED SURFACE AND ANOMALOUS ROUGHENING}

The surface growth model (3) is expected to generate scale-invariant surfaces with space-time correlations [10] obeying power-law scaling of the type

$$
\left\langle\overline{\langle h(\mathbf{x}, t)-h(\mathbf{x}+\mathbf{l}, t)]^{2}}\right\rangle^{1 / 2} \sim l^{\alpha} \mathcal{G}\left(l / t^{1 / z}\right),
$$

where the overbar denotes the average over all $\mathbf{x}$, the angular brackets denote the average over realizations, and the critical exponents $\alpha$ and $z$ are the roughening and dynamic exponents, respectively. The scaling function $\mathcal{G}(u)$ becomes constant for $u \ll 1$, and decays as $\sim u^{-\alpha}$ for $u \gg 1$.

As mentioned above, the model differs from the KPZ equation in that the Gaussian $\delta$-correlated noise term is replaced by a static columnar disorder (although also Gaussian and $\delta$-correlated). In physical terms this is not irrelevant, because it leads to long-range temporal correlations in the growth direction and the violation of the Galilean invariance, i.e., the transformation of variables $h^{\prime}=h+\boldsymbol{\epsilon} \cdot \mathbf{x}$ and $\mathbf{x}^{\prime}=\mathbf{x}$ $-\lambda \boldsymbol{\epsilon} t$ that leaves the KPZ equation unaltered. Geometrically this corresponds to an invariance under tilting of the interface by an infinitesimal angle $\boldsymbol{\epsilon}$. The most important consequence of Galilean invariance in the KPZ equation is that the nonlinear term $\lambda$ does not renormalize under the renormalization group (RG) transformation; in turn this implies the existence of an exact scaling relation $\alpha+z=2$. However, the static disorder in Eq. (3) breaks the Galilean invariance; hence $\lambda$ does renormalize and $\alpha+z \neq 2$

An earlier one-loop perturbative RG approach in Ref. [10] gave the critical exponents $z=2-(4-d)(2-d) /(12-5 d)$ and $\alpha=(4-d)^{2} /(12-5 d)$. In the following section, the RG approximation is compared with numerical simulations in $d$ $=1,2$. Note that the faceted patterns described in the last section cannot be captured by this standard RG analysis, which by definition is aimed at scale-invariant solutions. As will be shown in what follows, the pattern is also responsible for an anomalous roughening behavior of the surface. The reason for the failure of the standard scaling ansatz might be related to the fact that higher-loop corrections to the RG flow equations introduce infinitely many relevant terms of the form $\lambda_{2 n}(\nabla h)^{2 n}$, as has already been noticed in Ref. [10].

We now briefly review the concept of generic scaling since, as we shall see below, Eq. (3) exhibits anomalous scaling properties. Scale-invariant roughening and the corresponding power-law scaling of surface fluctuations, as cast in Eq. (5), is usually referred to as Family-Vicsek scaling $[18,19]$ and has been shown to be useful for a wide class of growth problems. However, over the last ten years, experimental studies in a variety of systems including growth of thin films, electrodeposition, fracture, or fluid imbibition [20-25], as well as theoretical studies [17,26-35], have confirmed the existence of the so-called anomalous kinetic roughening. The term anomalous actually refers to the observation that local and global fluctuations do exhibit scaling, but may do so with different exponents.

When looking at the scaling properties of scale-invariant growing surfaces, one can typically analyze the scaling behavior of local vs global fluctuations, which can show different scaling properties if anomalous scaling takes place. On the one hand, global fluctuations are typically measured by the dispersion of the surface around the mean value, so one calculates the global width $\left.W(L, t)=\overline{\left\langle[h(\mathbf{x}, t)-\bar{h}(t)]^{2}\right.}\right\rangle^{1 / 2}$, where the overbar denotes the average over all $\mathbf{x}$ in a system of lateral size $L$ and the angular brackets denote the average over realizations. For scale-invariant surfaces, one expects the global width to scale as $W(L, t) \sim L^{\alpha} \mathcal{G}\left(L / t^{1 / z}\right)$, where $\mathcal{G}$ is a scaling function with the same asymptotic behavior as in (5), and $\alpha$ is the global roughness exponent. Therefore, in the 
stationary regime $t \gg L^{z}$ we have $W_{\text {stat }}(L) \sim L^{\alpha}$.

On the other hand, one can also define a local roughness exponent $\alpha_{\text {loc }}$ from the scaling behavior of the local width $w(l, t)=\left\langle\left\langle\left[h(\mathbf{x}, t)-\langle h\rangle_{l}(t)\right]^{2}\right\rangle_{l}\right\rangle^{1 / 2}$, where $\langle\cdots\rangle_{l}$ denotes an average over $\mathbf{x}$ in a box window of lateral size $l$. For scaleinvariant surfaces local fluctuations are also expected to show scaling, $w(l, t) \sim l^{\alpha_{\mathrm{loc}} \mathcal{G}_{\mathrm{A}}}\left(l / t^{1 / z}\right)$, where the scaling function is now $\mathcal{G}_{\mathrm{A}}(u) \sim$ const for $u \ll 1$, and decays as $\sim u^{-\alpha_{\text {loc }} \text { for }}$ $u \gg 1$. This scaling behavior leads to a distinctive scaling with the window size in the stationary regime, so that $w_{\text {stat }}(l, L) \sim l^{\alpha_{\mathrm{loc}}} L^{\alpha-\alpha_{\mathrm{loc}}}$. It is worth noting the dependence of the stationary local width on the system size, which is a characteristic fingerprint of anomalous scaling. Standard scaling is recovered when $\alpha=\alpha_{\text {loc }}$, where there is no difference between the scaling properties of local and global fluctuations. On the contrary, anomalous scaling occurs when $\alpha \neq \alpha_{\text {loc }}$. This difference in the scaling local and global scaling has important implications in the form of the scaling functions, the meaning of the exponents, and their correct determination from experiments or simulations.

\section{A. Generic scaling ansatz}

Ramasco et al. [17] introduced the generic scaling ansatz in order to include all the existing scaling behaviors in scaleinvariant roughening in one single framework. They showed that all the different forms that dynamic scaling can take are naturally derived as subclasses of a more general scaling ansatz, whenever scale invariance of the correlation functions exists. Generic scaling is best formulated in Fourier space in terms of the structure factor (or spectral power spectrum) $S(k, t)=\langle\hat{h}(\mathbf{k}, t) \hat{h}(-\mathbf{k}, t)\rangle$, where $\hat{h}(\mathbf{k}, t)$ is the Fourier transform of the surface in a system of size $L, \hat{h}(\mathbf{k}, t)$ $=L^{-d / 2} \sum_{\mathbf{x}}[h(\mathbf{k}, t)-\bar{h}(t)] \exp (i \mathbf{k} \cdot \mathbf{x})$. Following [17], we write the ansatz

$$
S(k, t)=k^{-(2 \alpha+d)} s\left(k t^{1 / z}\right),
$$

where $\alpha$ is the the global roughness exponent and the scaling function takes the general form

$$
s(u) \sim \begin{cases}u^{2\left(\alpha-\alpha_{s}\right)} & \text { if } u \gg 1, \\ u^{2 \alpha+d} & \text { if } u \ll 1 .\end{cases}
$$

Here $\alpha_{s}$ is called the spectral roughness exponent. One can obtain the correlation functions in real space simply by integrating (6) over Fourier momenta $k=|\mathbf{k}|$. To be precise, the global width is given by $W^{2}(L, t)=\int(d k / 2 \pi) S(k, t)$, while one has that the local fluctuations are given by $w^{2}(l, t)$ $\propto \int_{2 \pi / L}^{\pi / a}(d k / 2 \pi)[1-\cos (k l)] S(k, t)$, where $a$ is the lattice spacing and $L$ is the system size. A caveat when performing the latter integral is that the various limits involved $(a \rightarrow 0$, $t^{1 / z} / L \rightarrow \infty$, and $\left.L \rightarrow \infty\right)$ may not commute [17,32]. This leads to different scaling behavior of $\mathcal{G}_{\mathrm{A}}(u)$ depending on the value of the exponent $\alpha_{s}$. This results in the following classification:

$$
\alpha_{s}<1 \Rightarrow \alpha_{\mathrm{loc}}=\alpha_{s}\left\{\begin{array}{l}
\alpha_{s}=\alpha \Rightarrow \text { Family-Vicsek } \\
\alpha_{s} \neq \alpha \Rightarrow \text { intrinsic; }
\end{array}\right.
$$

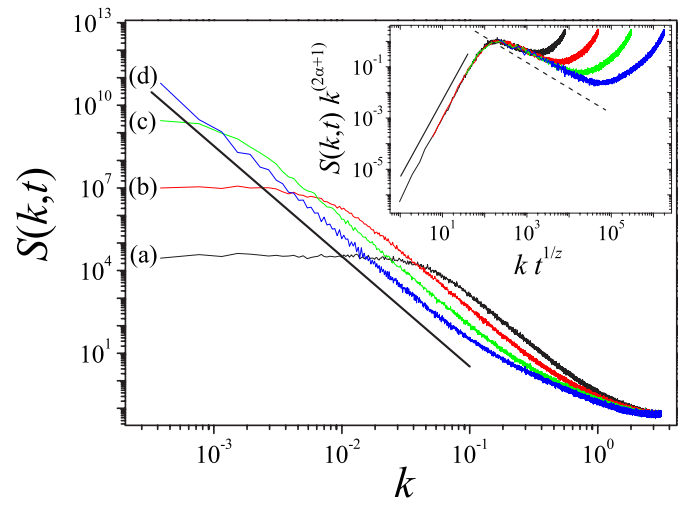

FIG. 3. (Color online) Structure factor of the surface obtained by numerical integration of (3) in systems of size $L=16384$. The curves $a, b, c$, and $d$ correspond to times $t / \Delta t=2^{13}, 2^{16}, 2^{19}$, and $2^{22}$, respectively. The straight line is plotted to guide the eyes and has slope -4 . Note the downward shift. The inset shows a data collapse according to the generic scaling form in Eqs. (6) and (7) with exponents $\alpha=1.07 \pm 0.05$ and $z=1.15 \pm 0.05$. The dashed and the solid straight lines have slopes -1 and 3 , respectively.

$$
\alpha_{s}>1 \Rightarrow \alpha_{\mathrm{loc}}=1\left\{\begin{array}{l}
\alpha_{s}=\alpha \Rightarrow \text { super-rough }, \\
\alpha_{s} \neq \alpha \Rightarrow \text { faceted }
\end{array}\right.
$$

Family-Vicsek scaling then corresponds to $\alpha=\alpha_{\mathrm{loc}}=\alpha_{s}<1$, and the interface is actually self-affine. However, dynamic scaling and power-law spacetime correlations are also possible for different settings than that of Family-Vicsek scaling, when the identity between different roughening exponents is not satisfied. In this case scaling is called anomalous. Intrinsic anomalous roughening and super-roughening have been shown [33-35] to be associated with a nontrivial power-law growth of the mean local slope $\left\langle\overline{(\nabla h)^{2}}\right\rangle$.

The scaling subclass we shall be interested in is the faceted anomalous scaling, since, as we have seen in Sec. II, solutions of Eq. (3) self-organize in rough faceted patterns. In Ref. [17] it was conjectured that the faceted subclass should be generic for rough faceted surfaces exhibiting power-law correlations.

\section{B. Faceted roughening in $1+1$ and $2+1$ dimensions}

According to the general framework just discussed in the previous section, a fluctuating faceted surface is expected to exhibit anomalous scaling properties as described by the "faceted" subclass in Eq. (8). We have computed the structure factor from a numerical integration of the surface growth model (3) in one and two dimensions. Generalization of the finite-difference scheme (4) for $d=2$ is straightforward.

In Fig. 3 we show the structure factor $S(k, t)$ of the surface in $d=1$ at times $t / \Delta t=2^{13}, 2^{16}, 2^{19}, 2^{22}$. Note that as time increases the curves shift downward, as expected from the generic scaling theory for $\alpha<\alpha_{s}$ (see previous section). To be precise, replacing Eq. (7) in (6), we have $S(k, t)$ $\sim k^{-\left(2 \alpha_{s}+d\right)} t^{2\left(\alpha-\alpha_{s}\right) / z}$ for $k t^{1 / z} \gg 1$, where the curves show a power-law displacement in time $t^{2\left(\alpha-\alpha_{s}\right) / z}$ that depends on the anomalous difference between the global and spectral rough- 


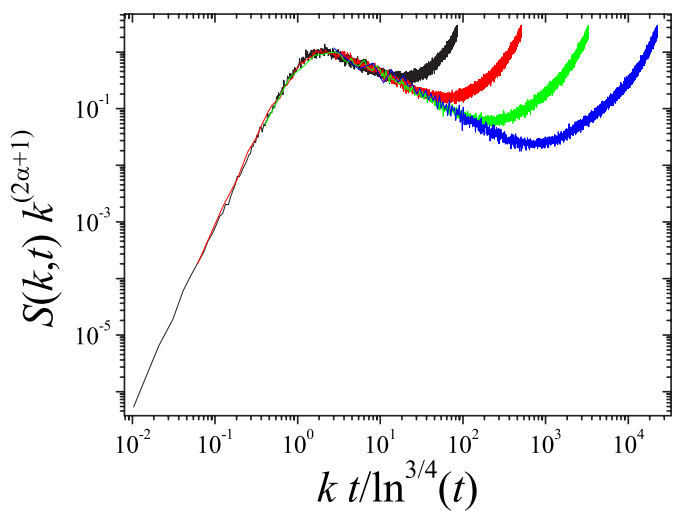

FIG. 4. (Color online) Collapse of the data presented in Fig. 3 using the $t \ln ^{-3 / 4} t$ subdiffusion law in (9).

ness exponents. Measuring the asymptotic decay of the structure factor curves, $k^{-\left(2 \alpha_{s}+d\right)}$, for long times and small momenta $k$ we immediately obtain an estimation for the spectral roughness exponent $\alpha_{s}=1.5 \pm 0.05$. The straight line in Fig. 3 is a guide to the eye and has a slope $2 \alpha_{s}+1=4$. According to the scaling ansatz (6) and (7) we can get a data collapse for $S(k, t)$. We find that the data collapse is optimal for the exponents $\alpha=1.07 \pm 0.05$ and $z=1.15 \pm 0.05$ and the result is shown in the inset of Fig. 3. Our results are in excellent agreement with the conjecture of Ref. [17] for rough faceted surfaces.

However, it is important to remark that the coarsening dynamics $\sim t / \ln ^{3 / 4} t$ strongly suggests that the exponent $z$ measured in this scaling analysis has to be just an effective exponent, which should be compared with the effective exponent measured in the coarsening analysis $z \approx z_{\text {coarse }}$. In Fig. 4 we show that in fact the data from Fig. 3 can be nicely collapsed according to

$$
S(k, t)=k^{-(2 \alpha+d)} s\left[k t / \ln ^{3 / 4}(t)\right],
$$

with $\alpha=1.07 \pm 0.05$. Nonetheless, one can consider the effective power law $t^{1 / z}$ as satisfactory in very good approximation (with logarithmic corrections). Note that this effective exponent will not be universal. Slightly different values can be observed for different systems following the same dynamics, depending, for example, on the disorder distribution [8].

We have also studied the growth model in $d=2$. In Fig. 5 we show the two-dimensional (2D) patterned surface at different times. The 1D triangles become cones due to the invariance of (3) under rotation of the two dimensional substrate. The structure factor of the surface in $d=2$ is shown in Fig. 6. Following the generic scaling functions Eqs. (6) and (7), we find $\alpha_{s}=1.00 \pm 0.02$ and a good data collapse is obtained for the exponents $\alpha=0.62 \pm 0.05$ and $z=1.20 \pm 0.05$ (see inset of Fig. 6). Results in $d=2$ are also in excellent agreement with the anomalous scaling ansatz for faceted surfaces in Eq. (8).

\section{LOCAL FLUCTUATIONS}

We now take a closer look at the surface to uncover some interesting properties of the model. For the sake of simplicity



FIG. 5. Numerical results in $2+1$ dimensions for a system of size $1024 \times 1024$ at times $t / \Delta t=2^{15}, 2^{16}, 2^{17}$, and $2^{18}$, from left to right. The gray scale plots show the $2 \mathrm{D}$ surface height (top panels) and the corresponding derivative in one substrate direction (bottom panels). Note the emergence and coarsening of a conical structure.

in the analysis, we restrict ourselves to the one-dimensional case, but the argument can be generalized to $2+1$ dimensions in a straightforward manner.

The basic observation is that at any given time the surface height can be seen as the sum of two different contributions. On the one hand, at large length scales the surface is faceted with a time-dependent average slope $\langle\bar{s}(t)\rangle$. The pattern can be constructed as a superposition of base functions of triangular shape, $h\left(x_{i}\right)-s\left|x-x_{i}^{c}\right|$, with cusps at certain sites $x_{i}^{c}$ and slopes $s$ randomly drawn from a (nonstationary) probability distribution peaked at $s_{\max }(t)$. On the other hand, at short wavelengths the surface exhibits fluctuations around the facet, thus locally deviating from the average global pattern.

We find that the facet component $h^{0}(x, t)$ and the shortscale noisy component $y(x, t)$ can be separated at any given time during the numerical integration as explicitly shown in Fig. 7 for a typical surface realization. Separation into these two components can be carried out numerically as follows. First, at any given time during the numerical simulation, the interface $h(x)$ is smoothed to remove local maxima and minima corresponding to local fluctuations upon the triangular structure. This is done by replacing the heights at every

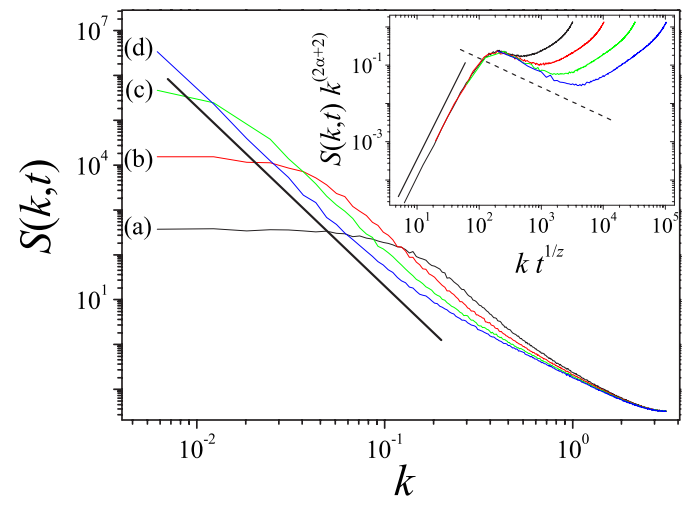

FIG. 6. (Color online) Structure factor of the 2D surface in a system of size $1024 \times 1024$. Curves $a, b, c$, and $d$ correspond to times $t / \Delta t=2^{12}, 2^{14}, 2^{16}$, and $2^{18}$, respectively. The straight line has slope $2 \alpha_{s}+2=-4$. In the inset a data collapse corresponding to the generic scaling ansatz, Eqs. (6) and (7), with exponents $\alpha$ $=0.62 \pm 0.05$ and $z=1.20 \pm 0.05$ is shown. The dashed and the solid straight line have slopes -0.76 and 3.24 , respectively. 


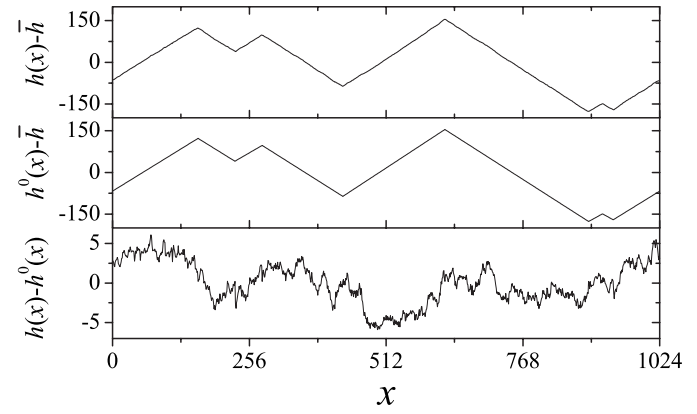

FIG. 7. Separation in pattern component $h^{0}(x)$ and local fluctuations $y(x) \equiv h(x)-h^{0}(x)$.

site by a spatial average over some arbitrary region around each site. Then, the locations of the cusp sites $x_{i}^{c}$ and valley sites $x_{i}^{v}$ of the smoothed curve are easily identified and the pattern $h^{0}(x)$ is defined as the set of straight lines connecting the cusp points $\left(x_{i}^{c}, h\left(x_{i}^{c}\right)\right)$ to the neighboring valley points $\left(x_{j}^{v}, h\left(x_{j}^{v}\right)\right)$. The interface corresponding to the fluctuations is then obtained by taking the difference $y(x)=h(x)-h^{0}(x)$.

The existence of these two different contributions makes it possible to express the surface height as

$$
h(x, t)=h^{0}(x, t)+y(x, t),
$$

where $h^{0}(x, t)$ is the facet component and $y(x, t)$ is the local fluctuation of the height around the global pattern component. One can think of the facet component as a superposition of base functions of triangular shape,

$$
h^{0}(x, t)=\sum_{i}\left[h\left(x_{i}\right)-s\left|x-x_{i}^{\mathrm{c}}\right| \Theta\left(x \mid x_{i-1}^{v}, x_{i}^{v}\right)\right],
$$

where the sum runs over the $N_{c}(t)$ substrate sites corresponding to the random location of the cusps, as shown in Fig. 7 for a typical evolution. The Heaviside-type function, $\Theta(x \mid u, v)=1$ if $u \leqslant x \leqslant v$ and 0 otherwise, defines the domain on the substrate interval $\left[x_{i-1}^{v}, x_{i}^{v}\right]$ between the nearest left, $x_{i-1}^{v}$, and right, $x_{i}^{v}$, shallow valley sites around every cusp site $x_{i}^{c}$.

The cusp sites $x_{i}^{c}$ and the valley sites $x_{i}^{v}$ are selected by the dynamics of the surface as the result of a competition between the particular realization of the static columnar disorder $\eta(x)$ and the KPZ nonlinearity, which forces the local surface slope to be bounded. Cusps tend to appear at strong depinning sites, where the columnar disorder is large and positive, and therefore growth is greatly enhanced at the expense of neighboring sites where the depinning force is lower. The cusp sites are uniformly distributed along the front and separated by a typical distance of the order of the coarsening length $\rho(t)$. The number of cusp sites (and the corresponding valleys) decreases rapidly in time due to coarsening, and one can estimate $N_{c}(t) \sim L / \rho(t) \sim L t^{-1} \ln ^{3 / 4} t$.

We find that the facet slopes $s$ are distributed according a certain probability density. As shown in Fig. 8, the probability density function $(\mathcal{P})$ for the facet slope, $\mathcal{P}\left(\nabla h^{0}\right)$, is a function peaked at $s_{\max }$. The distribution gets progressively narrower as it proceeds toward its stationary form. Estimates for the average slope time dependence of the facets have

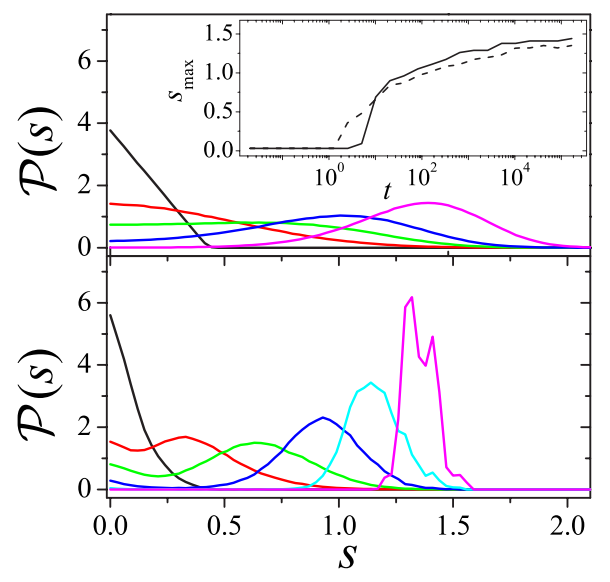

FIG. 8. (Color online) Probability density functions for the slopes of the whole interface (upper panel) and the facets (lower panel) at times $t / \Delta t=2^{1}, 2^{8}, 2^{10}, 2^{13}, 2^{24}$ and $t / \Delta t$ $=2^{1}, 2^{8}, 2^{10}, 2^{13}, 2^{17}, 2^{24}$, respectively. In both cases the most probable slope value increases with time. In the case of the facets the distribution also gets narrower in time. The inset shows the most probable slope values for complete interface (solid line) and the facets (dashed line), both tend to some maximum value in the longtime limit.

been given in [10]. In contrast, the slope distribution for the whole surface, $\mathcal{P}(\nabla h)$, is much wider, even at the stationary state. It is convenient to define the function $H(t)=\left\langle\overline{h^{0}(x, t)}\right\rangle$ as the average height of the facet component at time $t$, so that we have that the average interface velocity is $\left\langle\overline{\partial_{t} h}\right\rangle=\dot{H}(t)$ $+\left\langle\overline{\partial_{t} y}\right\rangle$, where $\left\langle\overline{\partial_{t} y}\right\rangle=\lambda\left\langle\overline{(\nabla y)^{2}}\right\rangle=c$ is a constant.

It is most remarkable that the pattern and local fluctuation components in Eq. (10) are completely decoupled. This can be used to obtain an equation for the dynamic evolution of the local fluctuation component $y(x, t)$, by adiabatic elimination of the pattern degrees of freedom $h^{0}(x, t)$. Decoupling of the two components can be proved as follows. According to Eq. (10) we have $\nabla h=\nabla h^{0}+\nabla y$, which leads to $\left\langle\overline{(\nabla h)^{2}}\right\rangle$ $=\left\langle\overline{\left(\nabla h^{0}\right)^{2}}\right\rangle+c / \lambda+2\left\langle\overline{\left(\nabla h^{0}\right) \cdot(\nabla y)}\right\rangle$, after space and disorder averaging. However, if pattern and local fluctuations are actually decoupled we have $\left\langle\overline{\left(\nabla h^{0}\right) \cdot(\nabla y)}\right\rangle=\left\langle\overline{\left(\nabla h^{0}\right)}\right\rangle \cdot\langle\overline{(\nabla y)}\rangle=0$, which is identically zero. Note that periodic boundary conditions imply that $\left\langle\overline{\left(\nabla h^{0}\right)}\right\rangle=\langle\overline{(\nabla y)}\rangle=0$ at all times. So if and only if the two processes are decoupled, we would expect

$$
\left\langle\overline{(\nabla h)^{2}}\right\rangle-\left\langle\overline{\left(\nabla h^{0}\right)^{2}}\right\rangle=c / \lambda
$$

to be satisfied at any time $t$. Indeed, numerical results in Fig. 9 show that the difference between the $\left\langle\overline{(\nabla h)^{2}}\right\rangle$ and $\left\langle\overline{\left(\nabla h^{0}\right)^{2}}\right\rangle$ curves tends to a constant as time increases, indicating that the pattern and local fluctuations get rapidly decoupled as the system evolves.

Decoupling of the global pattern and local fluctuation components is indeed a remarkable property, and allows us to get an evolution equation for the dynamics of the local fluctuations $y(x, t)$ within an adiabatic approximation. The surface degrees of freedom, i.e., the height field $h(x, t)$, are divided in Eq. (10) into facet and local fluctuation degrees of 


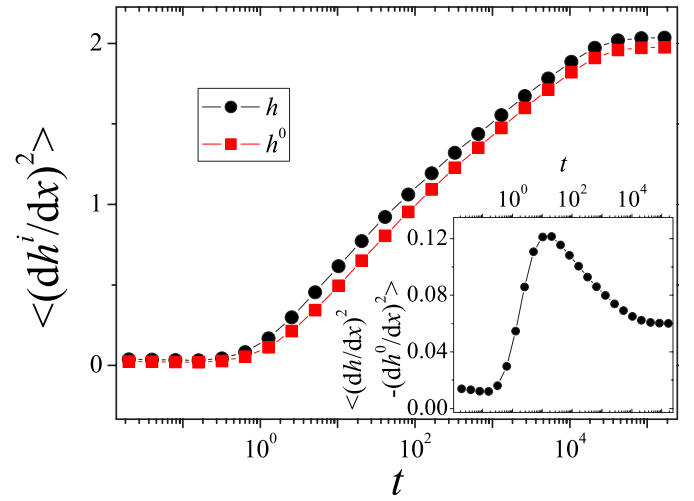

FIG. 9. (Color online) Average of the square of the spatial derivative of the whole interface $h(x, t)$ and the facets $h^{0}(x, t)$. In the inset the difference (12) is plotted. For large enough times, the difference tends to a constant value, confirming the decoupling of the local fluctuations from the pattern.

freedom. One can now integrate the fastest degrees of freedom, those corresponding to the global pattern, in order to arrive at an equation for the remaining variables, the field $y(x, t)$. Substituting the decomposition (10) in the surface growth evolution equation (3), we have

$$
\partial_{t} y=\nu \nabla^{2} y+\lambda(\nabla y)^{2}+f(x, t),
$$

where the stochastic driving field is $f(x, t)=-\partial_{t} h^{0}+\nu \nabla^{2} h^{0}$ $+\lambda\left(\nabla h^{0}\right)^{2}+2 \lambda\left(\nabla h^{0}\right) \cdot(\nabla y)+\eta(x)$. The approximation now amounts to replacing $f(x, t)$ by an effective noise $\widetilde{f}(x, t)$. One can think of the effective noise as an average of $f(x, t)$ over the dynamically faster degrees of freedom, i.e., $h^{0}$, which amounts to averaging over the long wavelengths. The decoupling between the two components, $\left\langle\left(\nabla h^{0}\right) \cdot(\nabla y)\right\rangle=0$, makes the multiplicative coupling term vanish, and we claim that the local fluctuation field is described by (13) with an effective noise $\widetilde{f}(x, t)$. In this approximation, the precise statistical properties of this effective noise term, which depend on the interplay between the static disorder, the random position of the cusps, and the slope selection discussed above, are neglected. These details are irrelevant for determining the dynamics of the local fluctuation $y$ as long as the effective noise $\widetilde{f}(x, t)$ probability distribution has finite moments and there are no long-range correlations. Under these general assumptions, one would conclude that the local fluctuations should belong to the KPZ universality class. This approximation turns out to be too rough though. In Fig. 10 we plot the structure factor of the noise component, $\langle\hat{y}(k, t) \hat{y}(-k, t)\rangle$, obtained from the decomposition (10) at time $t$. From data collapse we obtain a roughness exponent $\alpha=0.49 \pm 0.05$ and dynamic exponent $z=1.15 \pm 0.05$ for the local fluctuation component. These exponents are to be compared with KPZ critical behavior in $1+1$ dimensions, $\alpha_{\mathrm{KPZ}}=1 / 2$ and $z_{\mathrm{KPZ}}$ $=3 / 2$. Comparison reveals that this adiabatic approximation is too rough to give the correct value of the dynamic exponent of the local fluctuations, although it gives the correct spatial scaling with a roughness exponent $1 / 2$. Note that the value of $z$ coincides with that of the dynamic exponent of the

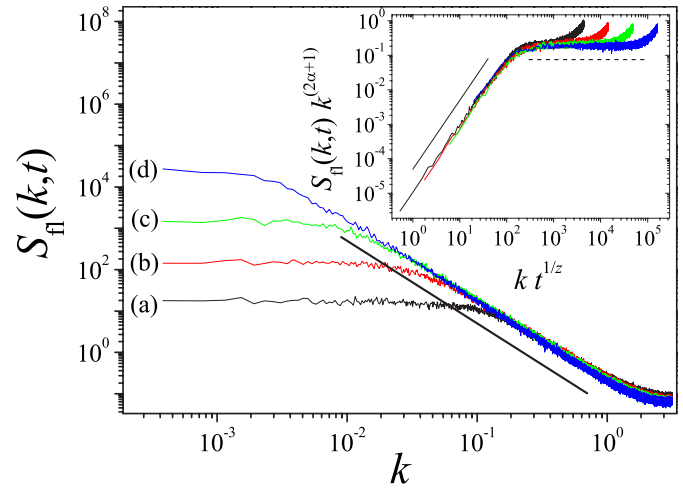

FIG. 10. (Color online) Structure factor of the local fluctuations $y(x, t)$ for a system of size $L=16384$ at times $t / \Delta t=2^{12}, 2^{14}, 2^{16}$, and $2^{18}$, for $a, b, c$, and $d$, respectively. The straight line is plotted to guide the eyes and has slope -2 . Inset shows a data collapse according to the Family-Vicsek subclass scaling. The best collapse was obtained for the exponents $\alpha=0.49 \pm 0.05$ and $z=1.15 \pm 0.05$. The dashed and the solid straight lines have slopes 0 and 2, respectively.

whole surface determined in Sec. III, as expected. We believe that the existence of long-range correlations in the effective noise $\tilde{f}(x, t)$ is responsible for this value of the dynamic exponent. Note that the effective noise term contains all the information about the dynamics of the global pattern, which should be far from being trivially described by a white noise term.

Finally, we find that the structure factor of the surface at any given time can also be obtained as the sum of the structure factors corresponding to the two components in Eq. (10),

$$
S(k, t)=\left\langle\hat{h}^{0}(k, t) \hat{h}^{0}(-k, t)\right\rangle+\langle\hat{y}(k, t) \hat{y}(-k, t)\rangle,
$$

which can be valid only whenever $\left\langle\hat{h}^{0}(k, t) \hat{y}(-k, t)\right\rangle$ is zero, so that there is no coupling between the pattern and the local fluctuation degrees of freedom. As shown in Fig. 11 simulation results fully support Eq. (14). Figure 11 shows the structure factor of the faceted pattern $h^{0}(x, t)$, the local fluctuations $y(x, t)$, and the complete interface $h(x, t)$ at two different times.

It becomes apparent that the anomalous scaling of the surface stems from that of the faceted structure. Since the vertical scale in Fig. 11 is logarithmic, the sum in (14) will essentially be dominated by the largest of the two, as one can easily see in Fig. 11. Therefore, the large-scale behavior of the complete interface is totally dominated by the scaling behavior of the facets. On the other hand, the structure factor $S(k, t)$ should cross over to that of the local fluctuations, $\langle\hat{y}(k, t) \hat{y}(-k, t)\rangle$, at short wavelengths. The structure factor of the facet component shifts downward with time because $\alpha$ $<\alpha_{s}$. This is not the case for the structure factor of the local fluctuations, which scale according to the Family-Vicsek ansatz where $\alpha=\alpha_{s}$. Thus the length scale at which the local fluctuations dominate the structure factor should grow with time. Whether the asymptotic scaling behavior of the facets can be observed in the limit $t \rightarrow \infty$ for a finite system depends 


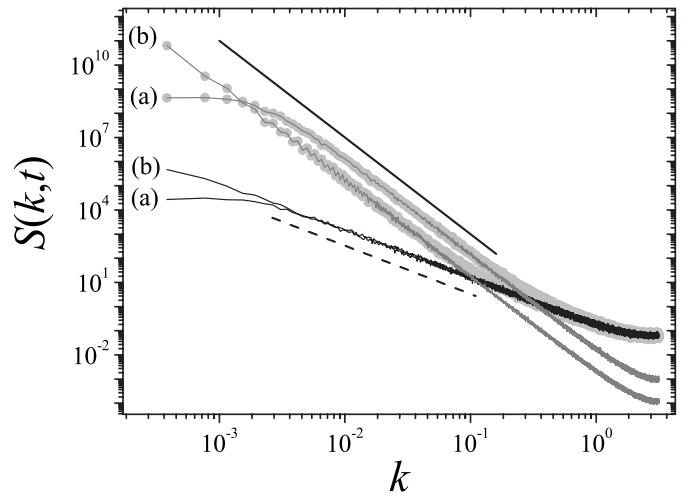

FIG. 11. Structure factor in a system of size $L=16384$. Numerical results for the facets (top solid curves $a$ and $b$ ), the local fluctuations (bottom solid curves $a$ and $b$ ), and the complete interface (solid circles) are shown at times $t / \Delta t=2^{18}$ and $t=2^{22}$, curves $a$ and $b$, respectively. It can be observed that the structure factor of the surface is dominated by the local fluctuations at short wavelengths, $k \gg 10^{-1}$ in the plot. However, the structure factor crosses over to that of the faceted pattern at long wavelengths, just as expected from Eq. (14). Therefore, the anomalous spectral roughness exponent (as well as the downward shift) of the complete interface arises from that of the facets. Unlike the structure factor of the facets, the local fluctuation power spectrum does not shift downward in time. The dashed and the solid straight lines are plotted to guide the eyes and have slopes of -2 and -4 , respectively.

on the relative strength of the local fluctuations. One could, for example, think of the case in which a thermal noise term is added to (3). If the noise strength is augmented, the crossover should shift to larger length scales; as a result, the asymptotic regime might not be detected if the system is finite.

\section{CONCLUSIONS}

We have studied a surface growth model whose deterministic part is that of the KPZ model but the thermal noise is replaced by a static columnar disorder $\eta(\mathbf{x})$. This model naturally arises from the simplest description of transport in trapping and amplifying disordered media though a HopfCole transformation, and it is one of the many equivalent mathematical formulations of the problem.

Numerical simulations in $1+1$ and $2+1$ dimensions revealed that the form of the solutions is far more complicated than what a one-loop RG calculation can produce. The surface organizes itself in facets of a certain characteristic slope. The slope dynamics is the result of an interplay between static disorder and the bounding effect of the KPZ nonlinearity. We have measured the coarsening length of the faceted structure, which in turn is equivalent to the motion of the the localization center in a multiplicative medium. Numerical estimates of the coarsening law are in good agreement with existing theoretical arguments $[8,10]$.

We have found that the existence of a faceted pattern leads to nontrivial scaling properties, including anomalous scaling. This is in excellent agreement with an earlier conjecture of Ramasco et al. for kinetic roughening of faceted surfaces. Moreover, we have found that the surface can be decomposed into two different contributions, the global pattern, which dominates the surface scaling at long wavelengths, and a local fluctuation component, whose spatial properties are characterized by a roughness exponent $\alpha$ $=\alpha_{\mathrm{KPZ}}$. An adiabatic approximation allowed us to relate the spatial scaling of the local noisy component to KPZ critical behavior. However, the dynamic exponent is dominated by the global pattern, and we found $z=1.15 \pm 0.05$ and $z$ $=1.20 \pm 0.05$ in $d=1$ and 2 , respectively. In the light of theoretical arguments $[8,10]$ that predict logarithmic corrections to the diffusion law, the value of the dynamic exponent $z$ has to be seen as an effective exponent. However, as we have shown, these corrections are very weak and extremely difficult to distinguish from purely power-law growth, even with three and a half decades of scaling.

As has been pointed out in Ref. [8], the scaling exponents may be dependent on the disorder distribution in this problem. This applies specially to the limit case of zero temperature (not studied in this paper), but some weaker effects have also been predicted for finite temperatures [which is the case more relevant to our study of (3)]. Indeed, we have carried out a full numerical study for a uniform disorder distribution and also found faceted growth with anomalous kinetic roughening (not shown). However, differences in the value of the dynamic exponent were obtained. This numerical difference is not surprising as $z$ is only expected to be an effective exponent. Moreover, the exact time dependence for the case with uniform disorder distribution may differ from $t / \mathrm{ln}^{3 / 4} t$, which was originally derived for Gaussian distributions of disorder.

In a more general context, we believe that similar mechanisms might be at work in other growth systems that produce kinetically rough faceted surfaces. In particular, our study suggests that the spectral roughness exponent that was conjectured by Ramasco et al. in Ref. [17] can play a role in the description of the scaling properties in other systems displaying this kind of patterned surface.

\section{ACKNOWLEDGMENT}

This work is supported by the DGI of the Ministerio de Educación y Ciencia (Spain) through Grant No. FIS200612253-C06-04. 
[1] T. Halpin-Healy and Y.-C. Zhang, Phys. Rep. 254, 215 (1995).

[2] Y.-C. Zhang, Phys. Rev. Lett. 56, 2113 (1986).

[3] W. Ebeling, A. Engel, B. Esser, and R. Feistel, J. Stat. Phys. 37, 369 (1984).

[4] A. Engel and W. Ebeling, Phys. Rev. Lett. 59, 1979 (1987).

[5] R. Tao, Phys. Rev. Lett. 61, 2405 (1998); 63, 2695 (1999); M. N. Rosenbluth, ibid. 63, 467 (1999); R. Tao, Phys. Rev. A 43, 5284 (1991); A. Valle, M. A. Rodríguez, and L. Pesquera, ibid. 43, 2070 (1991).

[6] Y.-C. Zhang, Phys. Rev. B 42, 4897 (1990); A. B. Kolomeisky and E. B. Kolomeisky, Phys. Rev. A 45, R5327 (1992).

[7] M. Kardar, G. Parisi, and Y.-C. Zhang, Phys. Rev. Lett. 56, 889 (1986).

[8] J. Krug and T. Halpin-Healy, J. Phys. I 3, 2179 (1993).

[9] M. E. Cates and R. C. Ball, J. Phys. (Paris) 49, 2009 (1988).

[10] T. Nattermann and W. Renz, Phys. Rev. A 40, 4675 (1989).

[11] D. R. Nelson and V. M. Vinokur, Phys. Rev. Lett. 68, 2398 (1992).

[12] I. F. Lyuksyutov, Europhys. Lett. 20, 273 (1992).

[13] T. Hwa, D. R. Nelson, and V. M. Vinokur, Phys. Rev. B 48, 1167 (1993).

[14] L.-H. Tang and I. F. Lyuksyutov, Phys. Rev. Lett. 71, 2745 (1993).

[15] I. Arsenin, T. Halpin-Healy, and J. Krug, Phys. Rev. E 49, R3561 (1994).

[16] L. Civale, A. D. Marwick, T. K. Worthington, M. A. Kirk, J. R. Thompson, L. Krusin-Elbaum, Y. Sun, J. R. Clem, and F. Holtzberg, Phys. Rev. Lett. 67, 648 (1991).

[17] J. J. Ramasco, J. M. López, and M. A. Rodríguez, Phys. Rev. Lett. 84, 2199 (2000).

[18] A.-L. Barabási and H. E. Stanley, Fractal Concepts in Surface Growth (Cambridge University Press, Cambridge, U.K.,
1995).

[19] F. Family and T. Vicsek, J. Phys. A 18, L75 (1985).

[20] H.-N. Yang, G.-C. Wang, and T.-M. Lu, Phys. Rev. Lett. 73, 2348 (1994).

[21] J. H. Jeffries, J.-K. Zuo, and M. M. Craig, Phys. Rev. Lett. 76, 4931 (1996).

[22] S. Huo and W. Schwarzacher, Phys. Rev. Lett. 86, 256 (2001).

[23] J. Santamaria, M. E. Gómez, J. L. Vicent, K. M. Krishnan, and I. K. Schuller, Phys. Rev. Lett. 89, 190601 (2002).

[24] J. M. López and J. Schmittbuhl, Phys. Rev. E 57, 6405 (1998); S. Morel, J. Schmittbuhl, J. M. López, and G. Valentin, ibid. 58, 6999 (1998).

[25] J. Soriano, J. J. Ramasco, M. A. Rodríguez, A. HernándezMachado, and J. Ortin, Phys. Rev. Lett. 89, 026102 (2002).

[26] J. G. Amar, P.-M. Lam, and F. Family, Phys. Rev. E 47, 3242 (1993).

[27] M. Schroeder et al., Europhys. Lett. 24, 563 (1993).

[28] S. Das Sarma, S. V. Ghaisas, and J. M. Kim, Phys. Rev. E 49, 122 (1994); S. Das Sarma, C. J. Lanczycki, R. Kotlyar, and S. V. Ghaisas, ibid. 53, 359 (1996).

[29] P. Smilauer and M. Kotrla, Phys. Rev. B 49, 5769 (1994).

[30] J. K. Bhattacharjee, S. Das Sarma, and R. Kotlyar, Phys. Rev. E 53, R1313 (1996).

[31] C. Dasgupta, S. Das Sarma, and J. M. Kim, Phys. Rev. E 54, R4552 (1996).

[32] J. M. López, M. A. Rodríguez, and R. Cuerno, Phys. Rev. E 56, 3993 (1997); Physica A 246, 329 (1997).

[33] J. Krug, Phys. Rev. Lett. 72, 2907 (1994).

[34] J. M. López, Phys. Rev. Lett. 83, 4594 (1999).

[35] J. M. López, M. Castro, and R. Gallego, Phys. Rev. Lett. 94, 166103 (2005). 